\title{
MiCROSTRUCTURAL SIMULATIONS \\ OF THE BONE SURROUNDING DENTAL IMPLANTS BY MEANS \\ OF A STOCHASTICALLY GENERATED FRAME MODEL
}

\author{
Éva Lakatos, Imre Bojtár \\ Department of Structural Mechanics, Budapest University of Technology and Economics \\ lakatoseva@mail.bme.hu
}

\begin{abstract}
The biomechanical behaviour of a dental implant plays an important role in its functional longevity inside the bone. Occlusal forces affect the bone surrounding an oral implant. To avoid fracture and bone resorption - by achieving the most even stress distribution in the bone implants should be applied that transfer occlusal forces to the bone within physiologic limits with geometry capable to enhance bone formation. The following study deals with the trabecular bone substance especially surrounding dental implants considering the microscopic conformation of the bone. The dental surgical use of a formerly developed stochastically generated frame model of the trabecular bone is introduced, which is created by interlinking a stochastically generated set of nodes in a certain domain, according to a previously defined linking-rule. The finite element model possesses the geometrical and mechanical microstructural properties - obtained from literature - of the trabecular bone substance of an average man from the edentulous mandibular region. The finite element beam model was submitted to compression, shear and torsion tests, and the macro-structural elastic properties were computed from the result data obtained by means of finite element analysis. The thus received frame model was combined with the finite element model of the cortical bone layer in the mandible (lower jaw bone) and a dental implant, where the incompleteness of the bone-implant interface was taken into account.
\end{abstract}

Keywords: dental implants; trabecular bone; finite element analysis; bone mechanics

\section{Introduction}

Application of dental implants is currently the most general and physiologically the most favourable procedure for tooth replacement in dental surgery. Implants can have either advantageous or destructive effect on the surrounding bone, depending on several physiological, material and mechanical factors. The most general method for estimating the biomechanical behaviour of the bone is finite element analysis, which has become indispensable in determining the mechanical behaviour - stress and strain distributions under a certain load - of the cortical and cancellous bone surrounding dental implants, since being applicable in vivo. These numerical experiments have their importance in making the implantation the possibly most secure, reliable and efficient, and the lifetime of the implant the longest conceivable, by finding the most favourable thread formation, surface, material, etc. In finite element analysis of the bone several assumptions need to be made, that influence the accuracy of the results. Recently, in most of the reported works the geometry of the implant can be modelled accurately, the assumptions are made in the aspect of the bone and bone- 
implant interface - where optimal osseointegration is assumed, which is acceptable after the bone healing period only at some parts of the implant surface - and the boundary conditions. Materials are mostly treated as continuum, with material properties usually set homogenous and linear with elastic material properties characterized by two material constants: Young's modulus and Poisson's ratio. This assumption approaches the behaviour of the metal alloys quite well, the properties of the bone are still intensively investigated.

The macromechanical properties of the bone are influenced by its microstructure. For this reason, microstructural information needs to be included in the analysis to predict individual mechanical bone properties ${ }^{1}$. Microstructural modelling of trabecular bone has become an extensively investigated field of biomechanical researches nowadays. The most commonly used tool for characterizing the complex architecture and material properties of bony organs is the conversion of micro-computed tomography images into micro-FE models ${ }^{2}$. The micro-computed tomography images (3D high resolution images) can be transformed into finite element models by means of different methods. The $3 \mathrm{D}$ reconstruction can be directly transformed into an equally shaped micro finite element model by simply converting all voxels to equally sized 8 -node brick elements (identical hexahedral elements). This results in finite element models with a very large number of elements, which means the need of high computational capacity and time ${ }^{3}$. Representing each trabecula with just one beam element reduces computational time and leads to the reduction in computational efforts as well ${ }^{4}$. These methods have been developed for simulating the mechanical behaviour of several types of bones of the human skeleton, usually for simulating the effect of bone diseases - such as osteoporo- sis - on the mechanical properties of the bone. In the majority of cases the models are from the vertebral or the femoral bone substance and not from the mandible or maxilla and include no implants in or cortical layer around the examined trabecular bone. The use of CTimaging means a serious restriction in the everyday applicability of the afore-mentioned bone models applying either volume- or beamelements. In micro-computed tomography imaging cadaveric samples are examined. In conventional CT-imaging or in Cone Beam Computed Tomography (CBCT) or Dental Volume Tomography (DVT) scanners, which are the latest generations of CT imaging in dentistry the - often otherwise healthy - patients are exposed to radiation exposure. On the contrary densitometry is accompanied by negligible radiation dose. Furthermore these kinds of examinations are patient specific and only suitable for describing the small, previously scanned fragment of the bone. In the initial phase of the researches the objective was to create a program that generates a stochastic beam structure, which has the format identical with the input data of program system ANSYS and has parameters, which are revisable according to the bone that is simulated (in the aspect of density, porosity, elastic properties etc.). A finite element bone model was produced, which - on the microstructural level - possesses the properties of the trabecular bone substance of an average man from the edentulous mandibular region, such as the geometry (average length and diameter) and the material attributes of a single trabecula or the porosity (or density) of the bone substance.

The received finite element frame model was submitted to compression, shear and torsion tests, and the macro-structural elastic properties were computed. The results were compared with the mechanical properties of the real bone tissue - drawn from literature - and the parameters of the model were set accord- 
ing to them ${ }^{5}$. In the following study the finite element model of the trabecular bone is combined with the models of the cortical layer of the mandible and of a screw type dental implant.

\section{Methods and results}

\subsection{Modelling the bone}

In building the finite element models of biological systems assumptions need to be made. In most reported studies - where the bone around dental implants is examined by means of finite element analysis - the macroscopic geometry of the bone is modelled and the assumption is made, that the materials are homogeneous and linear and that they have elastic material behaviour. In most models simulating the mechanical behaviour of bone surrounding dental implants the cortical and trabecular bone substances are treated as continuums - except for some early works, where sometimes only the compact bone is taken into account and the cancellous substance is neglected - with no local failures, discontinuities, cracks or inhomogeneities.

In case of the present study the porous structure of the trabecular bone was taken into account by using its previously published microstructural model, while the solid, compact material of the cortical bone was considered as a homogeneous, linearly elastic continuum.

The finite element beam model of the cancellous bone (Figure 1) was created by interlinking a stochastically generated set of nodes in a certain domain (Representative Volume Element), according to a previously defined linking-rule ${ }^{5}$. The extent of the Representative Volume is characteristic of the mechanical properties of the modelled material volume and has to be selected to be convenient to the aims of the planned investigations. In the present case the desired number of nodes was set in a $5 \mathrm{~mm} \times 5 \mathrm{~mm} \times 5 \mathrm{~mm}$ sized cubical shaped domain, which represents the identical sized bone substance. The stability of the model is provided by the appropriate number of linkings between the nodes and by the rigidity of the connections. Each node was connected with a beam to the closest at least three nodes, but considering the geometry of the trabecular bone of the human mandible this number had to be set from 5 to 7 . To achieve the required approximately $70 \%$ porosity (percentage value of voids volume of bone material in proportion to the volume of the whole cube) - according to literary data $6,7,8,9$ - the number of the nodes and the applied linkings were changed, with the length and diameter kept on the values achieved from microscopic images. In the thus received finite element model each trabecula was represented by one beam element. From the assortment of program system ANSYS the so-called BEAM188 element was applied, which is a three dimensional linear finite strain beam based on Timoshenko beam theory, in which shear deformation effects are included ${ }^{10}$. The consideration of the shear deformations are justified because of the beams being slightly stubby and possessing porous contexture.

The material properties of the trabeculae, and the macrostructural properties of the trabecular and the cortical bone were set according to the data achieved from the literature. Several techniques can be found for determining the Young's modulus of the single trabeculae ${ }^{6,11}$ : direct mechanical testing of a single trabecula in tension (Ryan and Williams, 1989), 3-point bending (Kuhn et al., 1987 and Choi et al., 1990) or buckling (Townsend et al., 1975 and Runkle and Pugh, 1975); ultrasonic wave propagation and scanning acoustic microscopy in trabecular bone specimens (Ashman and Rho, 
198812; Turner et al., 199913; and Nomura et al, 2007 ${ }^{14}$ ); finite element analysis (Pugh et al., 1973; Williams and Lewis, 1982; Mente and Lewis, 1987; van Rietbergen et al., 1995 and Kabel et al., 199915) and nanoidentation (Rho et al., 199716; Zysset et al., 199917). The macro structural Young's modulus value of cancellous bone was estimated by means of three different methods: using Hodgkinson and Currey's (1992) empirical equations relating modulus and density ${ }^{6}$, by the means of finite element analysis ${ }^{15}$ or compression tests ${ }^{18,19}$.

The finite element model of the cortical bone (Figure 1) was meshed with tetrahedral volume elements because of the complex geometry at the surface where the bone meets the implant.

\subsection{Modelling the implant}

Dental implants are artificial tooth root replacements. They are implanted in the jaw bones during small surgical operations to hold the prosthesis and to fasten it to the bone. In dental surgery the most commonly used implant types are the screw type endosteal - that are imbedded in the bone - implants, for this reason the most of the related studies - so do the present research of ours - examine the mechanical behaviour of these or of the bone surrounding them, considering various geometry, thread formation, length and diameter. Material properties of dental implants can be approached quite accurately - since being metal alloys - as homogeneous and linear with elastic material behaviour characterized by two material constants, Young's modulus and Poisson's ratio. Nowadays the application of metallic materials in dental implantology is limited to commercially pure titanium and its (Ti-6Al-4V) alloy ${ }^{20}$.

In this stage of the research a method was developed for describing the geometry of the screw type implants by means of mathematical functions and several modifiable or revisable parameters to make the procedure of finite element modelling faster and easier. The parameters, which can be altered according to the sizes and shapes of the implants chosen by the dental surgeon, are the following: the length of the implant, the inner and outer diameter of the implant, the thread formation and thread pitch, all of which can change along the length according to a function or differ in certain segments and the shape of the implant apex. In Figure 2. the models of two different dental implant types can be seen.

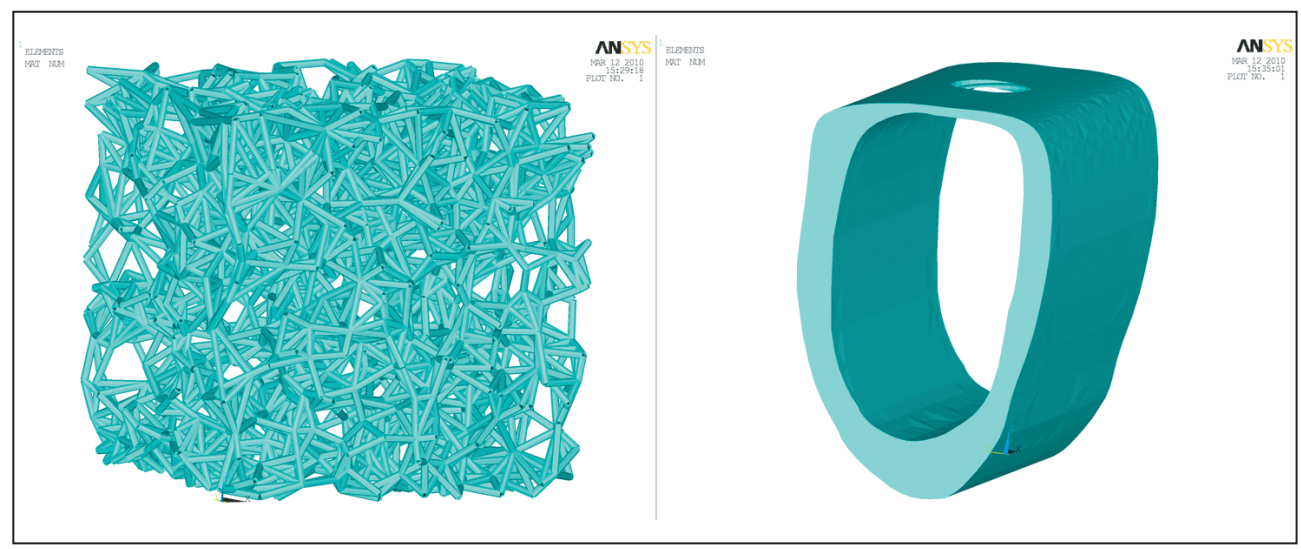

Figure 1. Finite element models of the trabecular (left) and the cortical (right) bone 


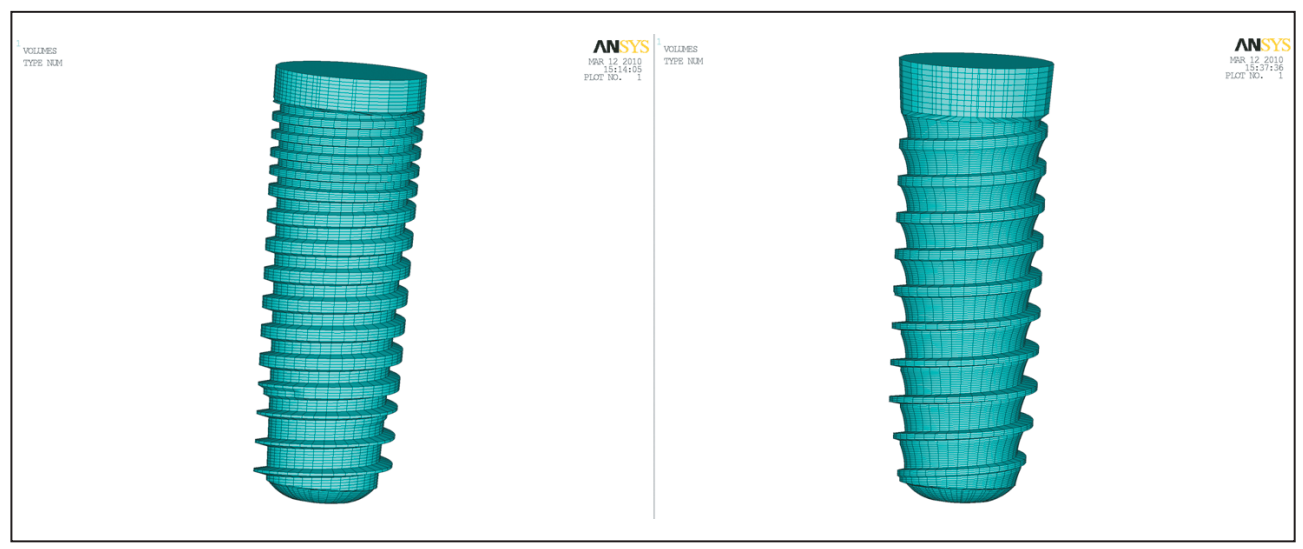

Figure 2. Finite element models of two different screw type dental implants

Since especially the behaviour of the bone is in the centre of our interest, and since being titanium alloy the material of the implants was assumed to be much stiffer than the bone, and only the surface of the screw was taken into account and meshed with triangular shell elements with high stiffness value.

\subsection{Modelling the bone-implant interface}

At the bone-implant interface most of the finite element models assume optimal osseointegration, which means, that the bone is perfectly bonded to the whole surface of the imp- lant, which cannot be assumed during the healing period and arguable after the complete healing, because of the presence of an intermediary layer between the two materials, which can be connected to the implant surface in different degrees. The degree of the osseointegration can be characterized by the BIC (boneimplant contact) value, which means the percentage of the implant area connected to the bone and the area of the whole implant surface $^{21}$.

The imperfection of the osseointegration could be taken into account by modelling the aforementioned intermediary layer as a mem-

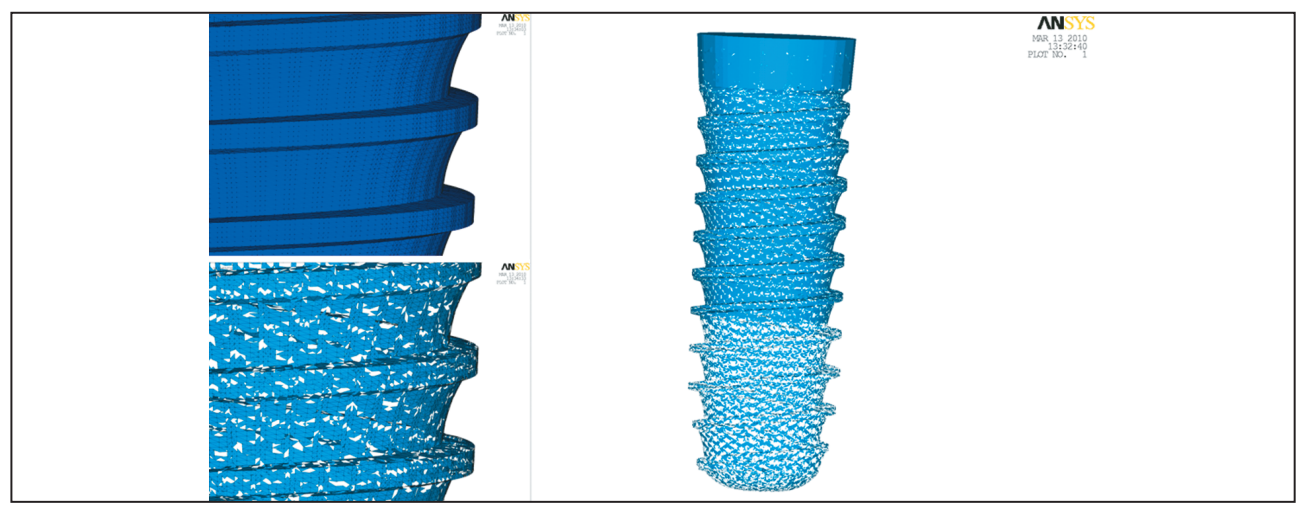

Figure 3. Finite element mesh of intermediary membrane between the bone and the implant with the finite element mesh of the implant 
brane covering different percents of the surface of the implant $(30 \%$ at the apex, $60 \%$ in the middle, where it meets the trabecular bone, and $90 \%$ at the upper region of the implant, where the mambrane meets the cortical bone) by using an additional layer also meshed with triangular shell elements (Figure 3). The elements forming the cortical and trabecular bone are attached to these elements instead of the implant.

\subsection{Compiling the model}

While compiling the complex finite element model from the aforementioned four parts (implant, trabecular bone, cortical bone, intermediary layer) especially while intersecting the beams with the volume or shell elements we faced several difficulties. The problem of connecting the different element types had to be solved. The beams cut by the surfaces of the implant or the cortical bone had to be connected to the nodes of the surface mesh (Figure 4), so an algorithm had to be developed, which changes the end points of the cut beams from the original connection points to the closest nodes of the surfaces. The second problem, which came up when the beams were cut, was the appearance of too short elements, and beams that were not connected to any other beams. These elements had to be found and erased.

\section{Conclusions}

In this study a numerical modelling method of the trabecular bone microstructure was introduced, which was developed for the examination of the mandibular or maxillar cancellous bone especially around dental implants in the framework of a former research. The mechanical behaviour of biological materials - such as cancellous bone - are most commonly examined by the means of direct mechanical testing or finite element analysis, the latter of which is the in vivo applicable method in humans. In the aspect of oral implantation in the upperand lower-jawbones the finite element models reported so far consider the trabecular bone substance as a continuum. The fact, that microstructural properties have remarkable effect on the overall behaviour of the bone, indicates the need of micromechanical simulations. The most commonly used method for modelling

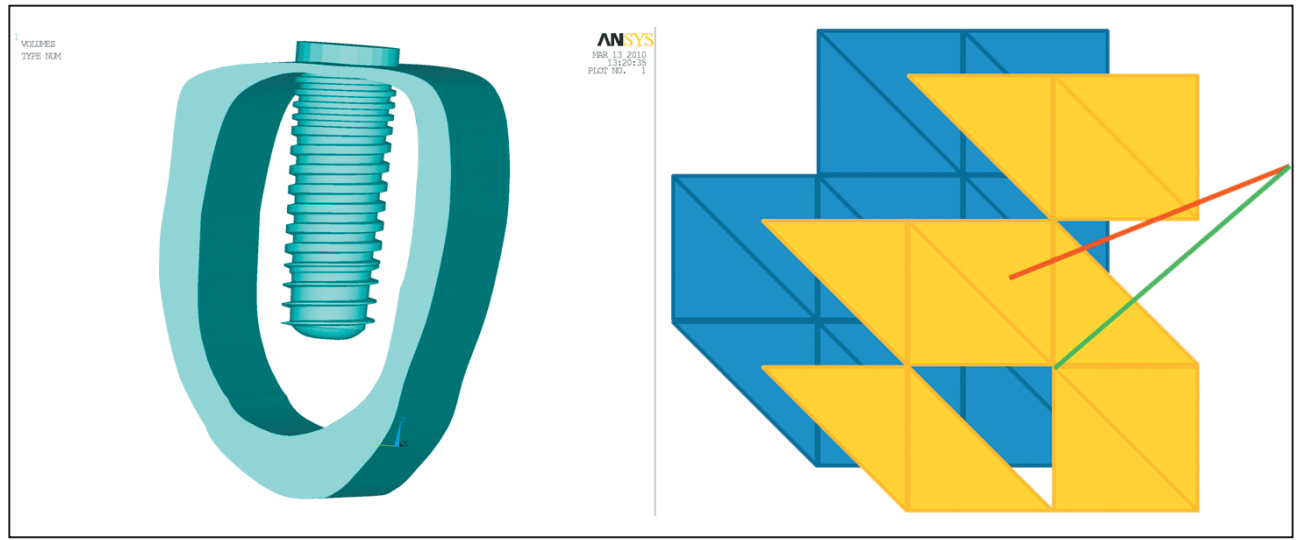

Figure 4. The combined finite element model of the implant and cortical bone (left) and the connection of the beams to the intermediary membrane (right), the original beam (red) changed to a properly connecting one (green) 
the cancellous bone's microstructure is the conversion of CT images into finite element meshes using either volume or beam elements, the latter of which overcomes the difficulties resulting from the high computational time and effort demand. The application of CT imaging can be avoided by creating a stochastically generated, porosity (or density) dependent finite element frame model, in which each trabecula is simulated by one beam ele- ment. The received model was combined with the finite element models of the mandibular cortical bone and the dental implant. The incomplete contact between the cancellous or cortical bone and the implant was taken into account by using an intermediary layer between the two surfaces, which covers the implant according to the value of the boneimplant contact area and connects it to the bone.

\section{REFERENCES}

1. Stauber $M$ and Müller R. Volumetric spatial decomposition of trabecular bone into rods and plates - a new method for local bone morphometry. Bone, 2006;38:475-484.

\section{Hellmich C, Kober C and Erdmann B. Microme-} chanics-Based Conversion of CT Data into Anisotropic Elasticity Tensors, Applied to FE Simulations of a Mandible. Annals of Biomedical Engineering. 2008;36(1):108-122.

3. Rudnyi EB, Van Rietbergen B and Korvik JG. Efficient harmonic simulation of a trabecular bone finite element model by the means of model reduction, Proceedings of $12^{\text {th }}$ Workshop "The Finite Element Method in Biomedical Engineering, Biomechanics and Related Fields". University of Ulm, July 2005., Proceedings of the $12^{\text {th }}$ FEM Workshop, 61-68.

4. Van Lenthe GH, Stauber M and Müller R. Specimen-specific beam models for fast and accurate prediction of human trabecular bone mechanical properties. Bone, 2006;39:1182-1189.

5. Lakatos É, Bojtár I. Stochastically generated finite element beam model for dental research. Periodica Polytechnica Ser Civ Eng 2009;53(1):3-8.

6. Gibson LJ and Ashby MF. Cellular solids - Structure and properties. University Press, Cambridge, second ed., 1997.

7. Renders GAP, Mulder L, Van Ruijven LJ and Van Eijden TMGJ. Porosity of human mandibular condylar bone. J. Anat., 2007;210(3):239-248.
8. Hogskinson R, Njehz CF, Whitehead MA and Langton CM. The non-linear relationship between BUA and porosity in cancellous bone. Phis. Med. Biol., 1996;40:2411-2420.

9. Moon HS, Won YY, Kim KD, Ruprecht A, Kim HJ, Kook HK and Chung MK. The threedimensional microstructure of the trabecular bone in the mandible. Surg. Radiol. Anat., 2004; $26 ; 466-473$.

\section{ANSYS 11.0 User's Manual}

11. Rho JY, Kuhn-Spearing $L$ and Zioupos $P$. Mechanical properties and the hierarchical structure of bone. Medical Engineering \& Physics, 1998;20:92-102.

12. Ashman $R B$ and Rho JY. Elastic modulus of trabecular bone material. Journal of Biomechanics, 1988;21(3):177-181.

13. Turner CH, Rho JY, Takano Y, Tsui TY and Pharr GM. The elastic properties of trabecular and cortical bone tissues are similar: results from two microscopic measurement techniques. Journal of Biomechanics, 1999;32:437441.

14. Nomura T, Katz JL, Powers MP and Saito C. A micromechanical elastic property study of trabecular bone in the human mandible. J. Mater. Sci.: Mater. Med. 2007;18:629-633.

15. Kabel J, Van Rietbergen B, Dalstra M, Odgaard A and Huiskes $R$. The role of an effective isotropic 
tissue modulus in the elastic properties of cancellous bone. Journal of Biomechanics, 1999; 32:673-680.

16. Rho JY, Tsui TY and Pharr GM. Elastic properties of human cortical and trabecular lamellar bone measured by nanoindentation. Biomaterials, 1997;18(20):1325-1330.

17. Zysset PK, Guo XE, Hoffler CE, Moore KE and Goldstein SA. Elastic modulus and hardness of cortical and trabecular bone lamellae measured by nanoindentation in the human femur. Journal of Biomechanics, 1999;32:1005-1012.

18. Kang $Q, A n Y H$ and Friedman RF. Mechanical properties and bone densities of canine trabecu- lar bone. Journal of Materials Science: Materials in Medicine, 1998;9:263-267.

19. O'Mahony AM, Williams JL, Katz JO and Spencer $P$. Anisotropic elastic properties of cancellous bone from a human edentulous mandible. Clinical Oral Implants Research, 2000; 11(5):415-421.

20. Divinyi T. Dental implantology (in Hungarian - Fogászati implantológia). Springer Hungarica Kiadó Kft., 1998.

21. Misch CE. Contemporary Implant Dentistry, Mosby Inc., $3^{\text {rd }}$ ed., 2007.

We thank Prof. Tamás Divinyi and Dr. Attila Szúcs from the Faculty of Dentistry, Semmelweis University, Budapest for providing the medical background for the research.

\section{Éva Lakatos}

Department of Structural Mechanics, Budapest University of Technology and Economics H-1111 Budapest, Múegyetem rkp. 3. 